\title{
Genetic diversity analysis using molecular marker in Terminalia chebula
}

\author{
T. N. Ranjini" , K. Bhanuprakash, ${ }^{1}$ M. A. Suryanarayana, ${ }^{2}$ and B. G. Yamuna ${ }^{3}$ \\ Indian Institute of Horticultural Research, Hessaraghatta, Bengaluru- 560089 (Karnataka ), INDIA \\ ${ }^{1}$ Section of Seed Sciences, India Institute of Horticultural Research, Hessaraghatta, Bengaluru - 56008 (Karnataka), \\ INDIA \\ ${ }^{2}$ Section of Medicinal plants, India Institute of Horticultural Research, Hessaraghatta, Bengaluru -560089 (Karnataka), \\ INDIA \\ ${ }^{3}$ Department of Agronomy, University of Agricultural and Horticultural Sciences, Shimoga-577204 (Karnataka), \\ INDIA \\ *Corresponding author: E-mail: ranjini.tantry@gmail.com
}

Received: February 3, 2015; Revised received: August 15, 2015; Accepted: December 9, 2015

Abstract: Terminalia chebula is an important medicinal plant, extensively used in Ayurveda, Unani and Homoeopathic medicines. The present study was aimed to reveal its genetic diversity based on molecular markers from twelve T. chebula accessions. Molecular diversity was studied using RAPD markers. A total of 8 polymorphic primers produced 314 polymorphic bands and 195 monomorphic bands. Unweighted Pair Group Method with Arithmetic Mean (UPMGA) dendrogram divided the accessions into 2 major clusters. Accession IIHRTc2 and IIHRTc10 showed maximum genetic diversity with $55 \%$ similarity. This characterization based on molecular markers will help in identification of economically useful accessions for further crop improvement programme.

Keywords: Dendrogram, Molecular Polymorphism, RAPD, Terminalia chebula

\section{INTRODUCTION}

Terminalia chebula (Combretaceae) commonly known as Indian Myrobalan, black Myrobalans in English and Harad in Hindi, indigenous to Pakistan and India. Among many Asian and African countries, is a popular folk medicine and it is well known for its homeostatic, laxative, diuretic, cardiotonic, antispasmodic, anti inflammatory, analgesic and wound healing properties (Kathirvel and Sujatha, 2012). It is one of the main constituent of Triphala ayurvedic drug, along with $T$. bellerica and Emblica officinalis (Naik et al., 2003). It is having high medicinal and economic properties and it is rich source of Non Wood Forest Product.

Over exploitation of Terminalia from different industries, in addition to the losses incurred by overgrazing, indiscriminate felling of trees, conversion of forest land into agriculture land and human settlements has led to a rapid depletion of genetic resources of Terminalia species. If timely steps are not taken for their conservation, cultivation and mass propagation, they may be lost from the natural habitat forever. Conservation of these resources alone cannot meet the ever increasing demand of pharmaceutical industry. It is, therefore, inevitable to develop genetically superior planting material by crop improvement for assured uniformity and desired quality and resort to organised cultivation to ensure the supply of raw material to the end users.
A better understanding of genetic diversity and its distribution is essential for its conservation and identification of superior planting materials for further crop improvement programme (Dangi et al., 2012).

Like any other crop, T. chebula also shows a large variation with respect to tree, fruit characters and phytoconstituent content. Most of the economic characters including yield are polygenically controlled and are highly influenced by environmental factors.

Presently, the classification of $T$. chebula is mainly based on morphological characters, but most of the times these characters are highly influenced by environmental conditions. Hence, the data obtained by such evaluations are not easily understood at genetic level, often resulting in maintenance of duplicate accessions or genotypes. Moreover, such evaluation studies demand higher cost and efforts.

Characterizing the genetic diversity existing in genotypes or accessions of a species is of prime importance to conserve existing variation and for crop improvement programme. In this regard, molecular and biochemical markers have an unique advantage to study the diversity. Therefore the present study was aimed to characterize the molecular diversity in twelve $T$. chebula accessions using Polymerase Chain Reaction (PCR) based marker such as Random Amplified Polymorphic DNA (RAPD) is considered powerful tools for assessing the existing genetic variation at the species, genus and population level. 


\section{MATERIALS AND METHODS}

Present investigation comprises of twelve T. chebula accessions, namely IIHRTc1, IIHRTc2, IIHRTc3, IIHRTc4, IIHRTc5, IIHRTc6, IIHRTc7, IIHRTc8, IIHRTc9, IIHRTc10, IIHRTc11 and IIHRTc12 located at Herbal garden, section of medicinal crops, Indian Institute of Horticultural Research, Hessaraghatta, Bengaluru.

\section{Molecular characterization}

Plant material: T. chebula leaf samples were collected from each accession. Total genomic DNA was extracted from young leaves following the standard CTAB method (Sarwat et al., 2010). The genomic DNA was quantified spectrophotometrically both at $260 \mathrm{~nm}$ and $280 \mathrm{~nm}$ wavelengths. The absorbance at $260 \mathrm{~nm}$ allows the calculation of DNA concentration in the sample. The extraction method yielded a good amount of DNA. The ratio of DNA to proteins ranged from 1.70 to 1.80 .

RAPD amplification: PCR amplification was carried out in $15 \mu \mathrm{l}$ reaction mixer containing $1.0 \mu \mathrm{l}$ of template DNA (30ng/ul), $2.0 \mu \mathrm{l}$ primer, $2.5 \mu \mathrm{l} 1 \mathrm{mM}$ dNTPs, $2.5 \mu \mathrm{l} 10 \mathrm{X}$ Taq buffer with $\mathrm{MgCl}_{2}$ and $6.7 \mu \mathrm{l}$ of sterile water. PCR reaction were performed in thermal cycler with an initial denaturation at $94^{\circ} \mathrm{C}$ for 4 min followed by 35 cycles at $94^{\circ} \mathrm{C}$ for $1 \mathrm{~min}, 37^{0} \mathrm{C}$ for $1 \mathrm{~min}$ and $72^{\circ} \mathrm{C}$ for 2 min with a final extension at $72^{\circ} \mathrm{C}$ for $7 \mathrm{~min}$. Amplified products were separated on $1.5 \%$ agarose gel in $1 \mathrm{X}$ TBE buffer by electrophoresis at $100 \mathrm{~V}$ and visualized with ethidium bromide staining in GelDoc System.

Analysed genetic diversity parameters such as poly- morphic information content (PIC), observed heterozygosity (Ho), effective multiplex ratio (EMR), marker index $(\mathrm{MI})$, primer resolving power $(\mathrm{Rp})$ and diversity index (DI) using RAPD primers.

PIC value was calculated using formula $P I C=2 f_{i}\left(1-f_{i}\right)$ Where $f_{i}$ is the frequency of the amplified allele and 1 $f_{i}$ is the frequency of null allele. Observed heterozygosity (Ho) was calculated by average of the (S) sum of bands present for each allele divided by (NC) number of genotypes under study $\left(\mathrm{Ho}=\sum \mathrm{S} / \mathrm{NC}\right)$ (Rolden Riuz et al.,2000). EMR was calculated by $E M R=n \times \beta$ $\left[\beta=n_{p} /\left(n_{p}+n_{n p}\right)\right]$, Where $n$ is the average number of bands amplified by accession to a specific system marker (multiplex ratio) and $\beta$ number of polymorphic loci $\left(n_{p}\right)$ and number of non-polymorphic loci $\left(n_{n p}\right)$ (Weir, 1996). Marker index is the product of polymorphic information content PIC and EMR (Powell et al., 1996). Rp was obtained according to Prevost and Wilkinson, (1999) $\mathrm{Rp}=\sum \mathrm{Ib}$, Where, Band informativeness (Ib) was calculted by $1-[2(0.5-\mathrm{p})]$ Where, 'P' is the proportion of accession containing the band. Diversity index is expected heterozygosity and was calculated according to Weir, (1996) as DI=1-1/L $\sum_{1}$ $\sum_{\mathrm{I}} \mathrm{Pi}_{2}$, Where, $\mathrm{Pi}$ is the frequency of $\mathrm{i}^{\mathrm{th}}$ allele at the 1 locus and $\mathrm{L}$ is the number of loci.

Statistical analysis: The RAPD bands were scored for its presence as ' 1 ' and absence as' 0 ' at each position are converted to binary matrix for analysis using numerical taxonomy and multivariate analysis system (NTSYS pc, ver. 2.02). The data were used to generate genetic similarity coefficient similarity matrix on the basis of jaccard's coefficient with SIMQUAL option.

Table 1. Variation in number of amplicons, percentage polymorphism due to different RAPD primers.

\begin{tabular}{|c|c|c|c|c|c|c|}
\hline S. N. & $\begin{array}{l}\text { Primer } \\
\text { code }\end{array}$ & Sequence & $\begin{array}{l}\text { Number of } \\
\text { polymorphic } \\
\text { amplicons }\end{array}$ & $\begin{array}{l}\text { Number of } \\
\text { monomorphic } \\
\text { amplicons }\end{array}$ & $\begin{array}{l}\text { Total number of } \\
\text { amplicons }\end{array}$ & $\begin{array}{l}\text { Percentage } \\
\text { polymorphism }\end{array}$ \\
\hline 1 & OPA3 & AGTCAGCCAG & 43 & 12 & 55 & 78 \\
\hline 2 & OPA10 & GTGATCGCAG & 63 & 12 & 75 & 84 \\
\hline 3 & OPB12 & CCTTGACGCA & 52 & 36 & 88 & 59 \\
\hline 4 & OPB17 & AGGGAACGAC & 39 & 24 & 63 & 61 \\
\hline 5 & OPC8 & TGGACCGGTG & 17 & 36 & 53 & 32 \\
\hline 6 & OPC20 & ACTTCGCCAC & 25 & 24 & 49 & 51 \\
\hline 7 & OPD19 & CTGGGGACTT & 22 & 24 & 46 & 47 \\
\hline 8 & OPE11 & GAGTCTCAGG & 53 & 0 & 53 & 100 \\
\hline Total & & & 314 & 195 & 482 & \\
\hline
\end{tabular}

Table 2. Genetic diversity parameters using RAPD primers in T. chebula accessions.

\begin{tabular}{|c|c|c|c|c|c|c|c|}
\hline $\begin{array}{l}\text { S. } \\
\text { N. }\end{array}$ & $\begin{array}{l}\text { Primer } \\
\text { code }\end{array}$ & $\begin{array}{l}\text { Polymorphic } \\
\text { Information } \\
\text { Content (PIC) }\end{array}$ & $\begin{array}{l}\text { Resolving } \\
\text { power (Rp) }\end{array}$ & $\begin{array}{l}\text { Effective Mul- } \\
\text { tiplex Ratio } \\
\text { (EMR) }\end{array}$ & $\begin{array}{l}\text { Diversity In- } \\
\operatorname{dex}(\mathrm{DI})\end{array}$ & $\begin{array}{l}\text { Marker } \\
\text { index (MI) }\end{array}$ & $\begin{array}{l}\text { Observed } \\
\text { Heterozy- } \\
\text { gosity (Ho) }\end{array}$ \\
\hline 1 & OPA3 & 0.34 & 4.50 & 6.13 & 0.43 & 2.62 & 0.76 \\
\hline 2 & OPA10 & 0.35 & 6.50 & 9.09 & 0.43 & 3.93 & 0.78 \\
\hline 3 & OPB12 & 0.46 & 0.83 & 3.00 & 0.42 & 0.42 & 0.99 \\
\hline 4 & OPB 17 & 0.29 & 3.83 & 4.50 & 0.34 & 1.55 & 0.63 \\
\hline 5 & OPC 8 & 0.27 & 1.34 & 1.80 & 0.92 & 0.92 & 0.30 \\
\hline 6 & OPC 20 & 0.24 & 1.83 & 2.67 & 0.32 & 0.85 & 0.56 \\
\hline 7 & OPD 19 & 0.17 & 2.67 & 5.44 & 0.57 & 3.13 & 0.74 \\
\hline 8 & OPE 11 & 0.41 & 3.17 & 7.00 & 0.37 & 2.58 & 0.78 \\
\hline
\end{tabular}


Table 3. Genetic similarity matrix (Jaccard's) of twelve T. chebula accessions.

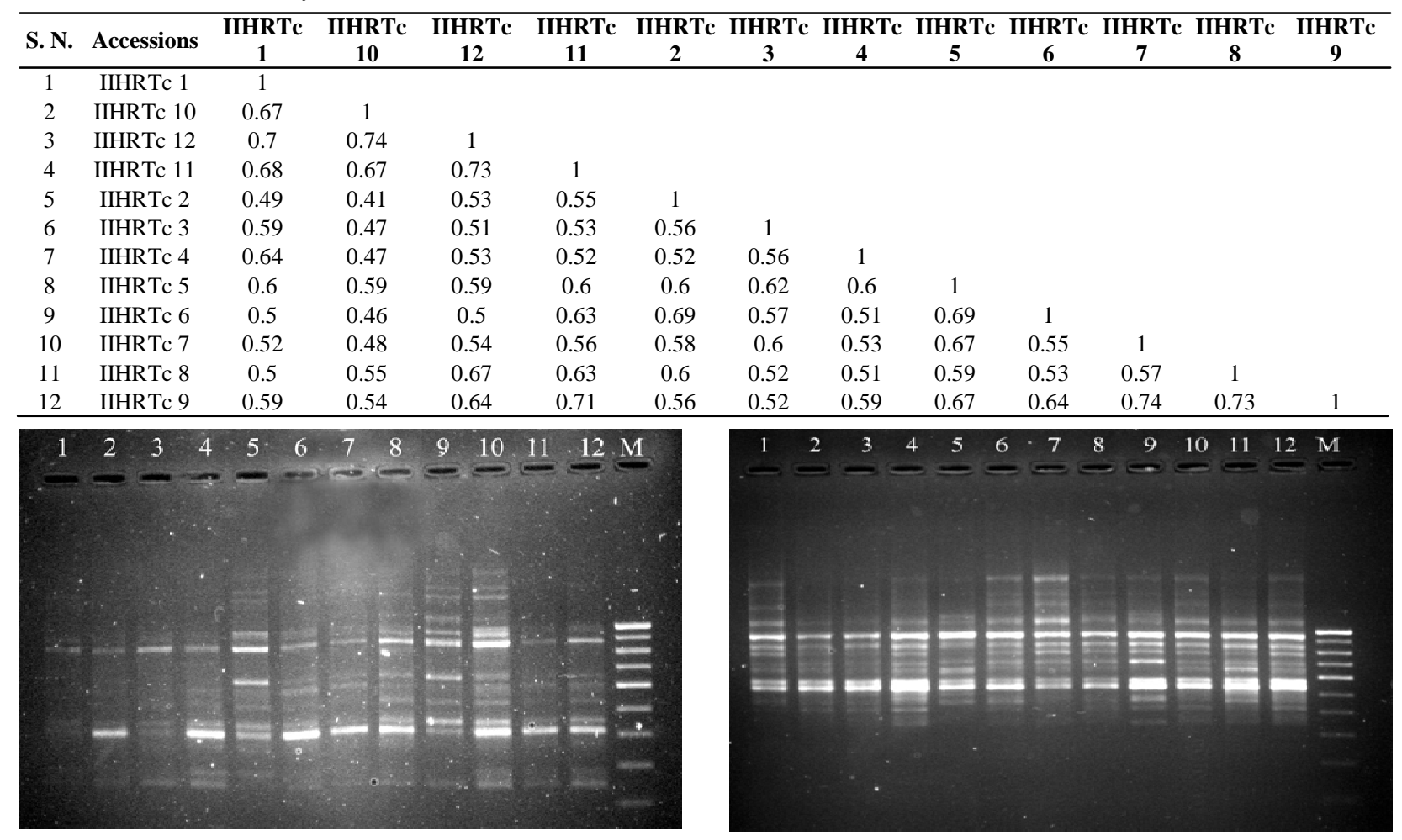

Fig. 1. RAPD profile of $T$. chebula accessions with primer OPA-10 and OPE-11.

(Lane 1: IIHRTc1, 2: IIHRTc2, 3: IIHRTc3, 4: IIHRTc4, 5: IIHRTc5, 6: IIHRTc6, 7: IIHRTc7, 8: IIHRTc8, 9: IIHRTc9, 10: IIHRTc10, 11: IIHRTc11, 12: IIHRTc12, M: 1 Kb ladder)

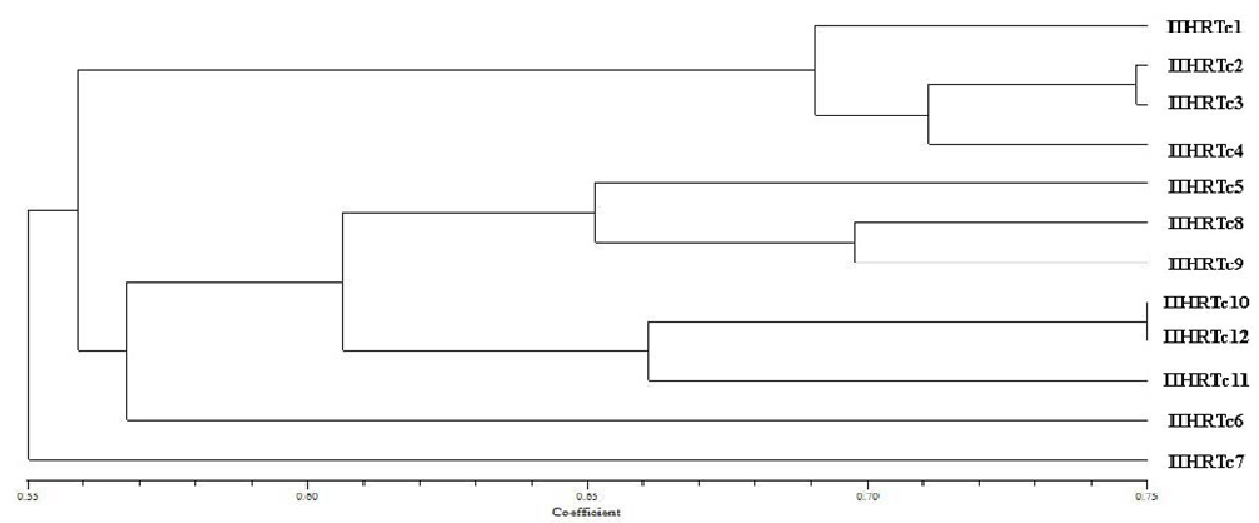

Fig. 2. Dendrogram showing the genetic relationship among T. chebula accessions.

The Dendrogram (cluster diagram) generated by unweighted pair group method with arithmetic average (UPGMA) algorithm using sequential agglomerative hierarchial and nested (SAHN).

\section{RESULTS AND DISCUSSION}

Overall, 40 RAPD primers were used to examine the extent of genetic diversity in 12 accessions. Amid the 40 RAPD primers, 8 primers gave polymorphic and reproducible banding pattern were chosen for further study.

Eight arbitrary oligoncleotide primers (OPA 3, OPA 10, OPB12, OPB 17, OPC 8, OPC 20, OPD 19 and
OPE 11) amplified 482 markers, out of which 314 were polymorphic. Each primer amplified on an average 24.3 polymorphic amplicons. The total number of amplicons produced by each primer ranged from 46 to 88 with an average of 60.25 amplicons per primer. Hundred per cent polymorphism was observed in primer OPE11 and least percentage of polymorphism was recorded in OPC8 primer (Table $1 \&$ Figure 1).

Genetic diversity parameters using RAPD primers in $T$. chebula accessions were calculated and presented in table 2. Polymorphic information content among markers ranged from 0.17 to 0.46 with mean 0.31 . The maximum PIC value was found in OPB 12 (0.46). The 
maximum $\mathrm{Rp}$ value was recorded in OPA10 (6.5). The maximum $\mathrm{Rp}$ value shows the usefulness of the primer OPA10 for distinguishing $T$. accessions. The maximum EMR value was seen in OPA10 (9.09). The maximum DI value was recorded for primer OPC8 (0.92). The highest MI value was found for primer, OPA10 (3.93). The primers with maximum MI value are more efficient. OPA10 primer with higher MI value is more efficient for distinguishing Terminalia accessions. The maximum observed heterozygosity (Ho) value was found for primer, OPB12 (0.99).

In order to analyze the relatedness among the accessions, a dendrogram was constructed on the basis of similarity matrix representing Jaccard's coefficient using the UPGMA algorithm. As evident from the dendrogram (Fig.2) 12 accessions formed 2 major clusters at $55 \%$ similarity. The accession IIHRTc7 is grouped separately from all the $T$. chebula accessions. Second major cluster (II) includes remaining eleven accessions. Accessions IIHRTc10 and IIHRTc12 are closely related at more than $75 \%$ similarity. Similar cluster analysis was obtained by Dangi et al. (2011) in 28 accessions of Terminalia bellerica based on DNA marker banding pattern.

The genetic similarity matrix generated by Jaccard's coefficient showed the extent of relatedness in $T$. chebula accessions. Ranged from 0.41 to 0.74 (Table 3), suggesting a very high genetic base among the $T$. chebula accessions. The highest genetic similarity of 0.74 was observed between IIHRTc10 and IIHRTc12. Results are in line with those obtained by Deshmukh et al. (2009) in five taxonomically critical Terminalia species and Sarwat et al. (2010) in Terminalia species, where genetic diversity among species were studied based on the result obtained by Jaccard's similarity matrix.

\section{Conclusion}

The RAPD analysis in the present study has proven to be useful in characterization and differentiation of $T$. chebula accessions. Large variation was observed among twelve accessions namely IIHRTc1 to IIHRTc12. Accessions IIHRTc10 and IIHRTc12 showed highest genetic similarity and IIHRTc7 has proven to be genetically diverse accession. This ge- netic diversity analysis study in T. chebula using molecular markers will assist in conservation and further improvement of the species through breeding. This study identified genetically diverse accessions and thus can help in further crop improvement programme.

\section{ACKNOWLEDGEMENT}

Thanks for Indian Institute of Horticultural Research, Hesseraghatta for providing necessary facilities required for the study.

\section{REFERENCES}

Dangi, B., Jain, R., Kachhwaha, S., Kothari, S.L. (2012). Assessment of diversity in Terminalia bellerica Roxb.using morphological, phytochemical and molecular markers. Nat. Acad. Sci. Lett. 35 (1): 135-144.

Deshmukh, V.P., Thakare, P.V., Chaudhari, U.S., Gawande, P.A. and Undal, V.S., (2009). Assessment of genetic diversity among Terminalia species using RAPD markers. Global J. Biotechnol.\& Biochem.,4 (2): 70-74.

Kathirvel, A. and Sujatha, V. (2012). In vitro assessment of antioxidant and antibacterial properties of Terminalia chebula Retz. leaves. Asian Pacific J. Tropical Biomedicine. 788-795.

Naik, G.H., Priyadarsini, K.I., Naik, D.B., Gangabhagirathi, R., Mohan, H. (2003). Studies on the aqueous extract of Terminalia chebula as a potent antioxidant and a probable radioprotector. Phytomedicine. 11:530-538.

Powell, W., Morgante, M., Andre, C., Hanafey, M. and Vogel, J., (1996). The comparision of RFLP, RAPD, AFLP, and SSR (microsatellite) markers for analysis, Mol. Breed.,2: 107-111.

Prevost, A. and Wilkinson, M.J., (1999). A new system for compairing PCR primers applied to ISSR fingerprinting of potato cultivars, Theor. Appl. Genet.,98: 107-112.

Roldan-ruiz, I., Dendauw J., Van, B.E., Depicker, A., De, L. M., (2000). AFLP markers reveal high polymorphic rates in ryegrasses (Lolium spp.). Mol. Breed.,6: 125134.

Sarwat, M., Das, S. and Srivastava, P.S. (2010). Estimation of genetic diversity and evaluation of relatedness through molecular markers among medicinally important trees: Terminalia arjuna, Terminalia chebula and, Terminalia bellerica. Mo.Biol.Rep. 2: 1121-1129.

WEIR, B. S., (1996). Genetic data analysis II: methods for discreate population genetic data, (Sinauer publishers, Sunderland MA, USA). 